\title{
Hipotiroidismo asociado a síndrome nefrótico en pacientes pediátricos del Centenario Hospital Miguel Hidalgo
}

\author{
Roque-Faz Carol Priscila*, Orozco-Loza Iraida Luz**, Barajas-Salcedo Gerardo**, Martínez-Gigena \\ María Pía**
}

\begin{abstract}
-
Resumen

- Introducción: en el síndrome nefrótico existe una pérdida de - proteínas de tamaño intermedio, así como también de otras

- proteínas como la globulina fijadora de tiroxina, transtire-

- tina y albúmina que conduce a la disminución de las hor-

- monas tiroideas y consecuentemente a un hipotiroidismo

- asociado. Objetivo: conocer la función tiroidea en los niños

- con síndrome nefrótico. Metodología: estudio observacional,

- descriptivo, prospectivo y longitudinal, universo de trabajo a

- pacientes pediátricos con diagnóstico de síndrome nefrótico

- de primera vez o con síndrome nefrótico que presenten re-

- caída, tratados previamente con prednisona o cualquier otro

- inmunosupresor. Resultados: se consultaron 57 pacientes

- con síndrome nefrótico en un periodo de 2 años. A 19 pa-

- cientes con nefrosis se les realizó perfil tiroideo, se encontró

- hipotiroidismo subclínico en 15 pacientes, con TSH de entre

- 4.95 y 10 mU/L; 2 pacientes presentaron niveles de TSH por

- arriba de $10 \mathrm{mU} / \mathrm{L}$ considerándose hipotiroidismo clínico y 2

- pacientes con niveles menores de $4.95 \mathrm{mU} / \mathrm{L}$ de dicha hor-

- mona considerándose dentro del rango normal. Conclusión:

- se observó que los pacientes pediátricos diagnosticados con

- síndrome nefrótico en la fase activa del mismo (nefrosis), al

- realizarles pruebas de función tiroidea, el 90\% presentan

- hipotiroidismo asociado, tomando en cuenta los niveles de

- la hormona estimulante de la tiroides. LUXMÉDICA AÑo 15,

NÚMERO 45, SEPTIEMBRE-DICIEMBRE 2020, PP 15-24.
\end{abstract}

Palabras clave: hipotiroidismo, síndrome nefrótico, hormonas tiroideas.

\section{Abstract}

Introduction: In nephrotic syndrome, there is a loss of intermediate-sized proteins and other proteins such as thyroxine-binding globulin, transthyretin, and albumin, leading to a decrease in thyroid hormones and consequently to hypothyroidism. Objective: To know the thyroid function in children with nephrotic syndrome. Methods: This is an observational, descriptive, prospective, and longitudinal study; it includes pediatric patients with a first-time diagnosis of nephrotic syndrome or patients who have relapsed and were previously treated with prednisone or any other immunosuppressant. Results: Fifty-seven patients with nephrotic syndrome were assisted in two years. A thyroid profile was performed in 19 patients with nephrosis. Subclinical hypothyroidism was found in 15 patients, with TSH between 4.95 and $10 \mathrm{mU} / \mathrm{L} ; 2$ patients had TSH levels $>10 \mathrm{mU} / \mathrm{L}$ classified as clinical hypothyroidism, and 2 patients had TSH levels $<4.95 \mathrm{mU} / \mathrm{L}$ considered within the normal range. Conclusion: In pediatric patients diagnosed with nephrotic syndrome in its active phase (nephrosis), $90 \%$ present associated hypothyroidism when a thyroid profile is performed considering thyroid-stimulating hormone levels. LUXMÉDICA AÑO 15, NÚMERO 45, SEPTIEMBREDICIEMBRE 2020, PP 15-24.

Residente del 3er año de la especialidad de pediatría en el Centenario Hospital Miguel Hidalgo. Aguascalientes, Ags. Orcid: 0000-0002-9940-4341 Correo electrónico hansonpris@hotmail.com

** Nefróloga Pediatra. Médico adscrito al servicio de Pediatría en el Centenario Hospital Miquel Hidalgo. Aguascalientes, Ags. Orcid: 0000-0002-15709317 Correo electrónico iraida_orozcoloza@yahoo.com.mx

*** Médico Pediatra. Médico adscrito al servicio de Pediatría. Centenario Hospital Miguel Hidalgo. Aguascalientes, Ags. Correo electrónico gerardobar2006@hotmail.com

**** Endocrinóloga Pediatra. Médico adscrito al servicio de Pediatría. Centenario Hospital Miguel Hidalgo. Aguascalientes, Ags. Correo electrónico piamgigena@hotmail.com

Fecha de recibido: 11 de marzo de 2020

Fecha de aceptación: 14 de julio de 2020

Correspondencia: Dra Iraida Luz Orozco Loza. Servicio de Pediatría. Centenario Hospital Miguel Hidalgo. Avenida Gómez Morín S/N La Estación, Alameda, Código postal 20259. Aguascalientes, Ags., México. Teléfono 4499946720 Correo electrónico iraida orozcoloza@yahoo.com.mx 


\section{Introducción}

El síndrome nefrótico (SN) es un trastorno renal causado por un conjunto de enfermedades, caracterizado por aumento en la permeabilidad de la pared capilar de los glomérulos renales que conlleva a la presencia de proteinuria, hipoproteinemia o hipoalbuminemia, ascitis $y$, en algunos casos, edema, hiperlipidemia e hipercoagulabilidad. ${ }^{1}$ Los hallazgos bioquímicos del SN se observan en la tabla 1.

El edema es el signo clínico más relevante. Su localización es variable según la actividad y posición del niño, pero predomina el edema maleolar, el pretibial y el palpebral; sin embargo, no es infrecuente encontrar también edema escrotal que puede dificultar la marcha del niño.

El síndrome nefrótico idiopático constituye el $90 \%$ de los SN en niños entre 2 y 12 años. La incidencia en población pediátrica menor de 16 años es de 2-7/100 000 niños por año, siendo la prevalencia 15 casos por 100000 niños. Se presenta preferentemente entre los 2 a 8 años, con máxima incidencia entre los 3 y 5 años. En niños es dos veces más frecuente en varones, diferencia que no existe en adolescentes y adultos. ${ }^{2}$

A nivel mundial, el síndrome nefrótico tiene una incidencia de 157 casos/100,000 niños. ${ }^{3}$ Es la glomerulopatía que se presenta con mayor frecuencia en niños y es uno de los diez síndromes más estudiados en nefrología. ${ }^{4}$ En México se desconoce la incidencia real. El Hospital Infantil de México "Federico Gómez" publicó en el 2014 que atienden, anualmente, 40 casos nuevos de síndrome nefrótico. ${ }^{5}$ En el estado de Aguascalientes no se ha publicado su incidencia. ${ }^{6}$ En el Centenario Hospital Miguel Hidalgo, en el año 2015, se registraron 1527 consultas en el servicio de nefrología, de las cuales 161 correspondieron a síndrome nefrótico, representando el $10.5 \%$ de las consultas. Además, en el mismo año se atendieron 12 casos nuevos. ${ }^{7}$ En el síndrome nefrótico hay pérdidas de proteínas tales como albúmina, transtiretina y globulina fijadora de tiroxina, conduciendo a la reducción de hormonas tiroideas y consecuentemente a un hipotiroidismo asociado. ${ }^{8}$ Hay estudios internacionales donde se ha demostrado la presencia de hipotiroidismo asociado en pacientes portadores de síndrome nefrótico. ${ }^{9-12}$ En México no hay nada publicado al respecto. ${ }^{6}$ En el Centenario Hospital Miguel Hidalgo no se solicitan de manera rutinaria las pruebas de función tiroidea en este grupo de pacientes. ${ }^{7}$

Desde hace mucho tiempo se ha estudiado la interacción de las hormonas tiroideas con el riñón. ${ }^{3}$ Las hormonas tiroideas (HT) son necesarias para el crecimiento y desarrollo del riñón y el mantenimiento del agua y la homeostasis de los electrolitos. Las hormonas tiroideas, tiroxina (T4) y triyodotironina (T3) tienen un rol importante en la maduración y desarrollo del esqueleto, afectan la calcificación endocondral y en todo el proceso de crecimiento del cartílago; ${ }^{4}$ por otra parte, el riñón está involucrado en el metabolismo y la 


\section{Tabla I}

Hallazgos bioquímicos del síndrome nefrótico

\begin{tabular}{|c|}
\hline Proteinuria masiva \\
\hline • Tres/cuatro cruces en tira reactiva \\
\hline - Cociente proteínas/creatinina en micción aislada $>3,5 \mathrm{mg} / \mathrm{mg}$ \\
\hline - Eliminación urinaria de proteínas $>40 \mathrm{mg} / \mathrm{m} 2 / \mathrm{h}$ o $>50 \mathrm{mg} / \mathrm{kg} /$ día \\
\hline Hipoalbuminemia $<\mathbf{2 , 5 - 3} \mathbf{g} / \mathbf{d l}$ \\
\hline Hipoproteinemia $<\mathbf{6 g} / \mathbf{d l}$ \\
\hline Otros datos habituales \\
\hline - Hiperlipemia: hipercolesteremia e hipertrigliceridemia \\
\hline - Hipercoagulabilidad \\
\hline
\end{tabular}

Tomada de Santos F. Síndrome nefrótico. Protocolos de nefrología. Boletín de la Sociedad de Pediatría de Asturias, Cantabria, Castilla 2006; 46(1): 19-23.

eliminación de las hormonas tiroideas..$^{5-6} \mathrm{La}$ fisiopatología del síndrome nefrótico se da por lesión del podocito que causa pérdida de la permeabilidad selectiva de la barrera de filtración glomerular al paso de las proteínas a través de la pared capilar glomerular lo cual conlleva a la presencia de niveles altos de proteína en la orina (proteinuria). ${ }^{2}$ Las hormonas tiroideas se unen a proteínas en la sangre, principalmente globulina de unión a tiroides, prealbúmina y albúmina. ${ }^{7}$ En el síndrome nefrótico (SN) hay pérdida de proteínas de tamaño intermedio (40-200 kDa) y las proteínas de unión a hormonas como la globulina fijadora de tiroxina (TBG), transtiretina y albúmina que conduce a la reducción de las hormonas tiroideas. ${ }^{9-10}$ Como resultado de esto, puede llevar al paciente a presentar hipotiroidismo subclínico o clínico asociado.

Mattoo TK mostró la existencia de un estado hipotiroideo en algunos niños con $\mathrm{SN}$, ellos recomendaron valoración de rutina de la función tiroidea y tratamiento de reemplazo temprano. ${ }^{11}$ Ito $S$ y col. mostraron evidencia de un hipotiroidismo leve en niños con SN no tratados, particularmente por pérdida urinaria de T4, T3, T4 libre, T3 libre y TBG. ${ }^{12}$ Sawant y col. encontraron que los pacientes con $\mathrm{SN}$ tienen un riesgo incrementado de hipotiroidismo subclínico. La función tiroidea vuelve a la normalidad cuando se resuelve la enfermedad no tiroidea. ${ }^{13}$ Gilles y col. Informaron que las anormalidades en la función tiroidea se observan en pacientes con proteinuria. En concreto, los niveles de TSH fueron mayores en los pacientes con enfermedades renales con proteinuria en comparación con los controles sin proteinuria. ${ }^{14}$ Iglesias en su estudio concluyó que el resultado de la proteinuria tuvo pérdida de la producción de la hormona estimulante de la tiroides.

La función tiroidea regresó a lo normal cuando se resolvió la nefrosis. ${ }^{14}$ Afroz S. y col. midieron el nivel de T3 y T4 en niños durante la nefrosis reportándolos como normal, en tanto que la TSH fue mayor a lo normal durante la nefrosis. Este estudio concluye que el síndrome nefrótico comúnmente tiene un hipotiroidismo leve o subclínico durante la proteinuria, aunque clínicamente son eutiroideos. ${ }^{15}$ Vidhi y col. compararon la función tiroidea durante la nefrosis y después del tratamiento con esteroide, encontrando elevación de la TSH 
durante la nefrosis, sin datos de hipotiroidismo; la función tiroidea se restablece cuando desaparece la nefrosis ${ }^{16}$.

Epstein informó la posibilidad de hipotiroidismo en pacientes con síndrome nefrótico en 1917. Sin embargo, los síntomas de hipotiroidismo con aumento de la hormona estimulante de la tiroides (TSH) en niños con síndrome nefrótico se descubrieron aproximadamente 30 años después. La excreción de albúmina renal no se compensa con el aumento de la producción de albúmina hepática en pacientes con síndrome nefrótico que conduce a una disminución del nivel de albúmina en la sangre. ${ }^{17}$

El objetivo de este estudio fue descartar hipotiroidismo asociado al síndrome nefrótico y en caso de presentarlo determinar la frecuencia de hipotiroidismo subclínico y clínico en los pacientes pediátricos con síndrome nefrótico en su fase activa del Centenario Hospital Miguel Hidalgo.

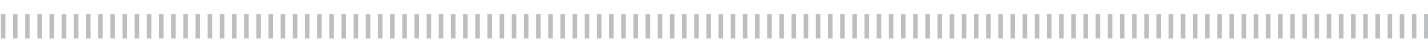

\section{Material y métodos}

Estudio observacional, descriptivo, prospectivo y longitudinal, en donde el universo de trabajo fueron pacientes pediátricos del Centenario Hospital Miguel Hidalgo portadores de síndrome nefrótico tanto diagnosticados por primera vez como los que presenten recaída durante el periodo del 1 de junio del 2016 al 1 junio del 2018.

Se incluyeron pacientes pediátricos con síndrome nefrótico con diagnóstico de primera vez, con síndrome nefrótico que presenten recaída, tratados previamente con prednisona o cualquier otro inmunosupresor. Se excluyeron pacientes con hipotiroidismo congénito, tumor pituitario, agenesia de tiroides, enfermedad de Graves, hipotiroidismo previo al síndrome nefrótico. Se consideró hipotiroidismo subclínico con niveles de TSH de $4.95 \mathrm{uUl} / \mathrm{m}$ a 9.99 $\mathrm{uUl} / \mathrm{m}$ e hipotiroidismo clínico con niveles de $\mathrm{TSH}$ mayores de $10 \mathrm{uUl} / \mathrm{m}$. El instrumento de recolección fue un cuadro en donde se encontraban datos para diagnosticar la fase activa del síndrome nefrótico y resultados del perfil tiroideo del paciente los cuales fueron: edema, albúmina sérica, colesterol, triglicéridos, TSH, T3 Total, T3 Libre, T4 Total, T4 Libre. El perfil tiroideo fue realizado en un equipo de sistema de inmunodiagnóstico VITROS 3600. Se utilizó el programa estadístico IBM SPSS Statistic 20.0 y el programa STATISTICA 8.0. Los procedimientos necesarios para nuestro estudio estuvieron sujetos a las normas éticas manejadas en el código de Núremberg, informe de Belmont y declaración de Helsinki que, en resumen, contienen los siguientes puntos importantes: respeto por las personas, beneficencia y justicia, respetando el reglamento de la Ley General de Salud, por lo que a pesar de ser un estudio observacional contamos con un consentimiento informado para los padres o tutores de los niños que participaron en el estudio.

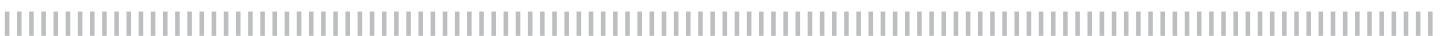

\section{Resultados}

Se consultaron 57 pacientes con síndrome nefrótico en el servicio de nefrología pediátrica dentro del periodo correspondiente a dos años, de los cuales, a 19 pacientes se les diagnosticó nefrosis, correspondiendo el $33 \%$ del total (figura 1 ).
Se observó que, de los 19 pacientes en la fase activa del síndrome nefrótico (nefrosis), al realizarles el perfil tiroideo, 17 de ellos presentaron niveles de hormona estimulante de la tiroides (TSH) por arriba del rango normal, considerándose hipotiroidismo asociado al síndrome nefrótico, siendo 
el $90 \%$ de los pacientes analizados (figura 2). De acuerdo con los niveles de la TSH, se encontró a 15 pacientes (78\%) con hipotiroidismo subclínico (TSH 4.95 - $10 \mathrm{mU} / \mathrm{L}$ ); dos pacientes $(10.5 \%)$ con hipotiroidismo clínico (TSH superior a $10 \mathrm{mU} / \mathrm{L}$ ); y dos pacientes $(10.5 \%$ ) eutiroideos (niveles de TSH menores de $4.95 \mathrm{mU} / \mathrm{L}$ (figura 3).

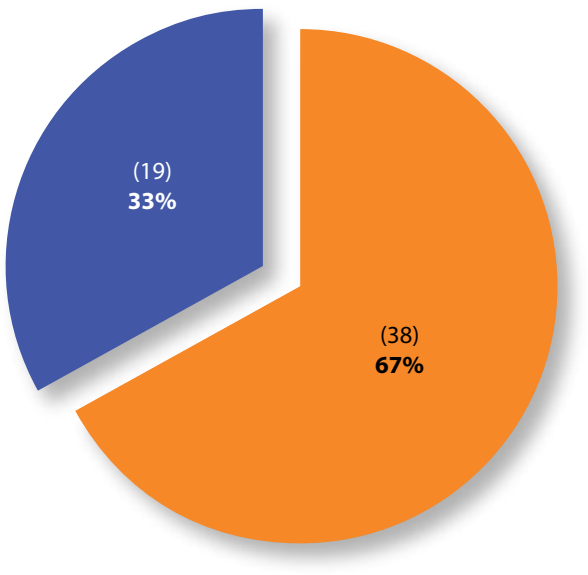

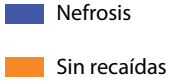

Figura 1. Consultas totales de síndrome nefrótico con el porcentaje de niños con nefrosis y niños sin recaídas.

\section{Hipotiroidismo asociado a síndrome} nefrótico $n=19$

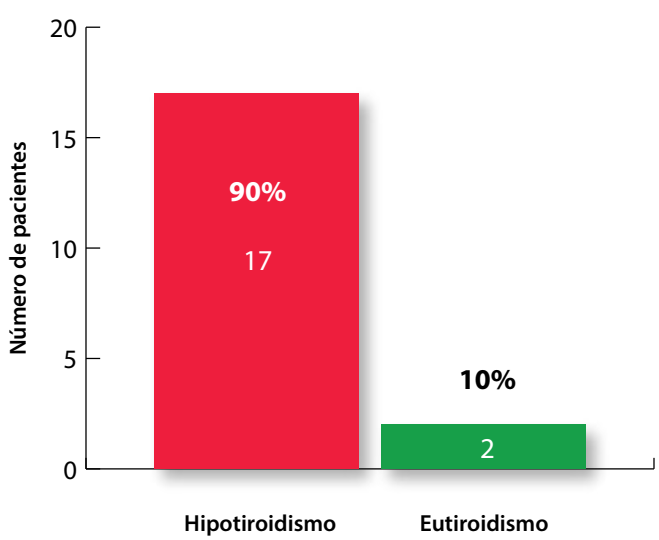

Figura 2. Porcentaje de pacientes con hipotiroidismo de los diagnosticados con síndrome nefrótico.

A los pacientes con hipotiroidismo al momento de la nefrosis, se les vuelve a solicitar perfil tiroideo, donde presentan una disminución de la misma hormona al momento de la remisión de la fase activa del síndrome nefrótico (figura 4). Cabe mencionar que en 4 de los 19 pacientes se documentó una

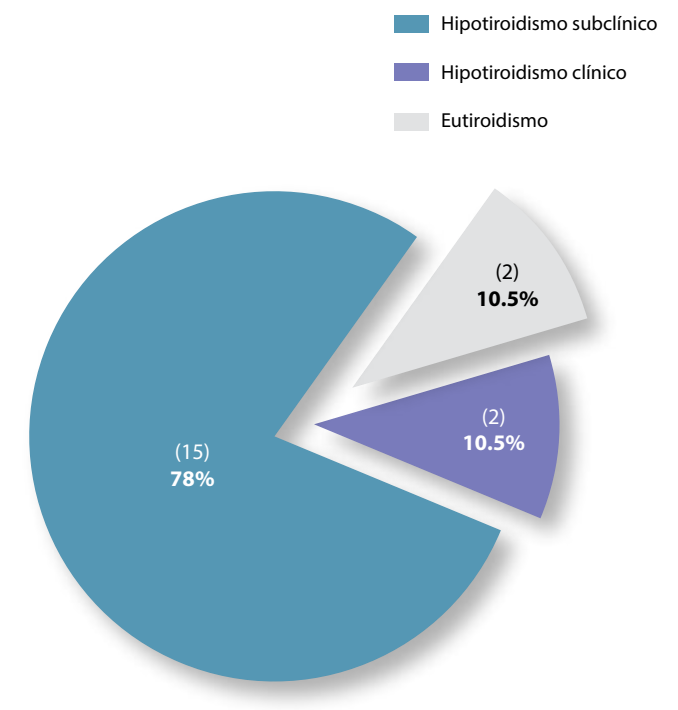

Figura 3. Porcentaje de pacientes con hipotiroidismo subclínico y clínico asociado a síndrome nefrótico.

segunda recaída a través del perfil tiroideo con reporte de TSH superior a $5 \mathrm{uUl} / \mathrm{ml}$. En la figura 3 se representan los niveles de TSH de los 23 eventos (19 pacientes y cuatro que presentaron recaída) en el momento de la nefrosis y la remisión, observándose que, en la nefrosis, la mayoría de los pacientes se 
encuentran con niveles de TSH por arriba la remisión, los niveles disminuyen a rangos de los rangos normales $y$, al momento de normales.

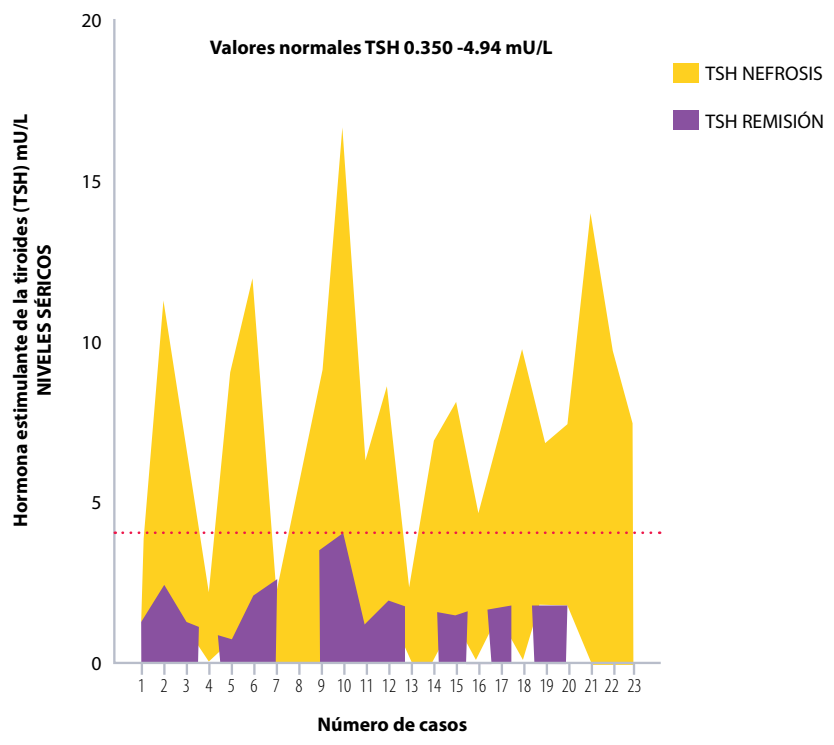

Figura 4. Niveles séricos de la TSH del número de casos total en el momento de la fase activa del síndrome nefrótico (nefrosis) y la remisión.

Las edades de los pacientes incluidos hasta los 13 años, con una media de 5.68 en el estudio correspondieron desde 1 año años (figura 5).

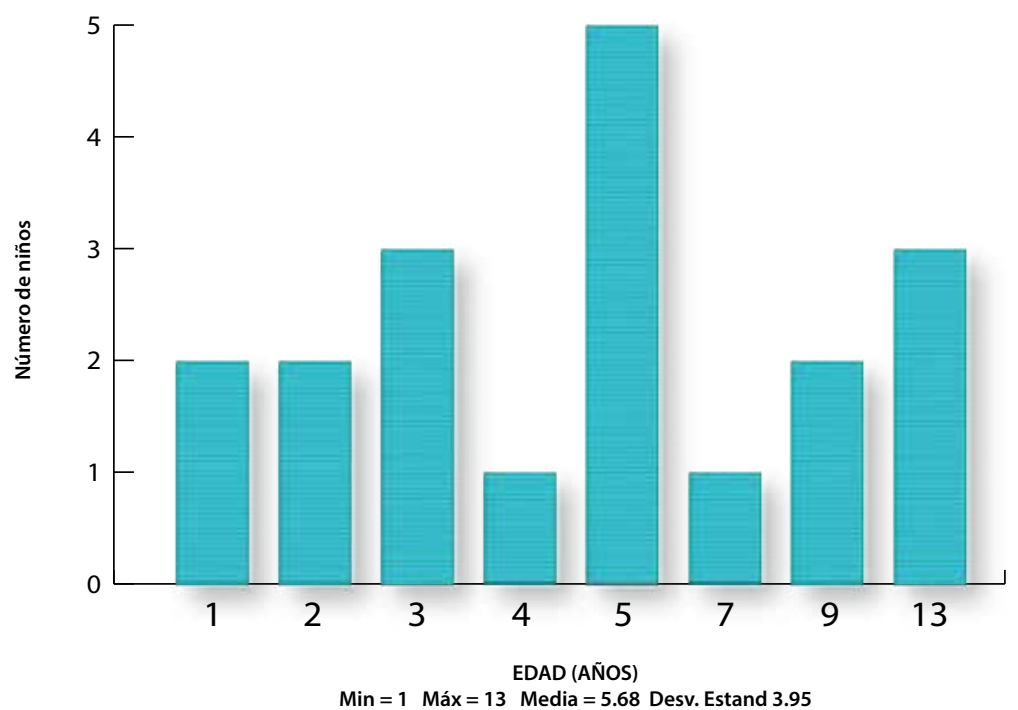

Figura 5. Presentación por edad de los pacientes que presentan síndrome nefrótico en su fase activa. 
El sexo predominante de los pacientes niños y 6 niñas correspondiendo el $68 \%$ y en el estudio fue el masculino, siendo 13 32\% respectivamente (figura 6). PRESENTACIÓN POR SEXO

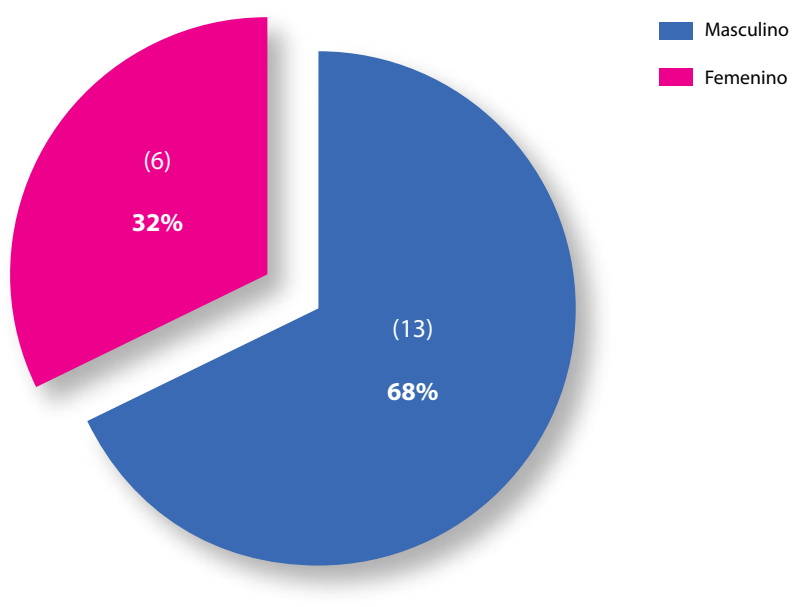

Figura 6. Presentación por sexo de los pacientes que presentan síndrome nefrótico en su fase activa.

\section{Discusión}

En el periodo comprendido de junio de 2016 a junio de 2018 se consultaron 57 niños con diagnóstico de síndrome nefrótico en el servicio de Nefrología Pediátrica en el Centenario Hospital Miguel Hidalgo, de los cuales, las edades correspondieron desde 1 hasta 13 años, lo que se relaciona con la literatura de Román Ortiz, ${ }^{2}$ ya que se documenta que el $90 \%$ del SN en niños se encuentra entre los 2 y 12 años y que se presenta preferentemente entre 2-8 años, con máxima incidencia entre los 3-5 años, siendo que en este estudio la desviación estándar es de 3.95 años y la media de 5.68 años, similar a lo ya reportado en la literatura mencionada.

En relación con el sexo, la bibliografía de Román Ortiz, ${ }^{2}$ al igual que en la bibliografía de Halty M. ${ }^{18}$ y Fernández E. S., ${ }^{19}$ refieren que hay más predisposición de pa- decer síndrome nefrótico en hombres que en mujeres y en este trabajo se observó que el $68 \%$ de los pacientes en cuestión son del sexo masculino y el $32 \%$ son del sexo femenino, dato que también es similar a la literatura.

Fueron 19 pacientes que se encontraron en fase activa del síndrome nefrótico, por lo que se les realizó perfil tiroideo antes de dar manejo correspondiente, en donde se observó que en 17 pacientes la TSH se reporta por arriba de $5 \mathrm{uUl} / \mathrm{ml}$, es decir, superior a rangos normales de la misma. En un estudio realizado por Afroz y cols. se demostró que 21 niños de 85 con síndrome nefrótico, con ataque inicial o recaída, a quienes se les realizó perfil tiroideo, tuvieron niveles de TSH por arriba de los valores normales, ${ }^{15}$ siendo el $24 \%$. En comparación con este estudio, obtuvimos un número menor de pacientes con recaída o 
ataque inicial; sin embargo, el $90 \%$ de los 19 pacientes (en fase activa del síndrome nefrótico) se presentó con niveles de TSH más arriba de lo normal. Después de $8 \mathrm{se}$ manas de tratamiento, se les solicita laboratorios para demostrar la remisión de la fase activa del síndrome nefrótico, confirmando en el $100 \%$ de los pacientes su remisión, por lo que se les solicita un nuevo perfil tiroideo. Sólo 14 pacientes se presentaron a la consulta con reporte del segundo perfil tiroideo, ya que 2 de ellos ya no regresaron a la consulta de nefrología pediátrica y 3 ya no se los realizaron. Los 14 pacientes en su segundo perfil tiroideo reportan niveles de la TSH dentro de los rangos normales, esto quiere decir que, en la remisión de la fase activa del síndrome nefrótico, el hipotiroidismo desaparece, demostrando que se asocia al síndrome nefrótico. En 4 de los 19 pacientes se documentó una segunda recaída de la cual se realizó el perfil tiroideo correspondiente a la nefrosis, en los cuales también se reporta TSH superior a $5 \mathrm{uUl} / \mathrm{ml}$, siendo también que en el segundo perfil tiroideo se remite el hipotiroidismo asociado en la remisión de la nefrosis, coincidiendo con el estudio realizado por Afroz y cols. donde también menciona que los niveles de TSH al momento de la remisión de la fase activa del síndrome nefrótico regresan a lo normal, considerándose un hipotiroidismo temporal mejorando con la remisión ${ }^{15}$.

Encontramos que dos pacientes con hipotiroidismo, es decir, con niveles de TSH superiores a $10 \mathrm{uUl} / \mathrm{ml}$, sin datos clínicos de hipotiroidismo, se enviaron al servicio de endocrinología pediátrica y actualmente se encuentran en seguimiento tanto por el servicio de endocrinología como de nefrología pediátrica.

No existe correlación de factores de riesgo en los dos pacientes que presentaron hipotiroidismo clínico en nuestro estudio. Sin embargo, uno de ellos presentó varías recaídas del síndrome nefrótico. En las últimas dos recaídas correspondientes al periodo de nuestro estudio, al momento de realizarle el perfil tiroideo, se observó que ha ido aumentando progresivamente la TSH en cada recaída, siendo en la última mayor de $10(14.13 \mathrm{uUl} / \mathrm{ml})$, y una lesión renal focal y segmentaria, corticorresistente. El otro paciente con lesión renal de cambios mínimos presentó sólo una recaída en el periodo de nuestro estudio, en la cual se le realizó perfil tiroideo con TSH mayor de $10(13 \mathrm{uUl} / \mathrm{ml})$, la cual, al momento de la remisión del síndrome nefrótico, disminuyó a rangos normales. Ambos pacientes tienen una edad de 5 años. Al momento de la nefrosis a ambos pacientes se les encontró edema de predominio facial y genital a consecuencia del síndrome nefrótico, sin presentar alguna característica clínica de hipotiroidismo clínico.

Kacer y cols. reportan un caso de un niño con síndrome nefrótico congénito e hipotiroidismo que tenía un alto requerimiento de tiroxina (100-150 mg/d) desde la infancia hasta los 4 años, al que se le realizó una nefrectomía bilateral; sin embargo, el hipotiroidismo persistió, pero el requerimiento de tiroxina fue disminuyendo, ${ }^{20}$ por lo que se asume que los pacientes pueden presentar hipotiroidismo en el que se necesite tratamiento sustitutivo hormonal así como seguimiento por el servicio de endocrinología. ${ }^{21}$ Además, es importante mencionar que tuvimos 2 casos con eutiroidismo en la fase activa del síndrome nefrótico. En el estudio de Sharma S. y cols donde se realiza un estudio de 50 niños con síndrome nefrótico a los cuales se les realizó pruebas de función tiroidea en su fase activa, el $80 \%$ no presentaron hipotiroidismo asociado y $20 \%$ si lo presentaron de los cuales el $70 \%$ tienen hipotiroidismo subclínico y el $30 \%$ hipotiroidismo manifiesto, ${ }^{22}$ con respecto a nuestro estudio se observó el 10\% de pacientes eutiroideos y el $90 \%$ con hipotiroidismo asociado con lo que difiere del estudio anteriormente mencionado; sin embargo, coincide en que en este estudio encontramos más de la mitad de los pa- 
cientes con hipotiroidismo siendo más subclínico que clínico; añadimos que coincide con el estudio mencionado que el hipotiroidismo subclínico en síndrome nefrótico es temporal y puede mejorar con la remisión. Sin embargo, la proteinuria prolongada en pacientes con síndrome nefrótico puede provocar un daño progresivo de los túbulos renales y una absorción deficiente de proteínas de bajo peso molecular (LMW) que pueden agotar aún más la reserva tiroidea y conducir a un hipotiroidismo manifiesto. Por lo tanto, el examen de la tiroides se debe recomendar rutinariamente en estos niños. ${ }^{23}$

Con respecto a las hormonas tiroideas, se observó que en los 23 eventos totales de este estudio, en su fase activa del síndrome nefrótico, la T3 y T4 libres se encontraron en rangos normales más frecuentemente que el resto de las hormonas tiroideas, en el estudio de Afroz y cols refieren que el valor medio de T3 en suero $(0,65 \pm 0,31$ $\mathrm{ng} / \mathrm{ml})$ y $\mathrm{T} 4(5,04 \pm 4,18 \mathrm{?g} / \mathrm{ml})$ en 85 niños nefróticos durante la nefrosis estuvo dentro del límite normal, ${ }^{15}$ datos que coinciden con los del presente estudio. Además se observó que la T4 total es la que con mayor frecuencia se presenta en niveles por debajo del rango normal de esta hormona, coincidiendo con el estudio de Ebadi A. y cols., quienes realizan un estudio de 20 niños con síndrome nefrótico a los cuales se les realiza pruebas de función tiroidea y observaron que los niveles de T4 fueron significativamente bajos en el síndrome nefrótico en comparación con las demás hormonas. ${ }^{23}$ También se observó que, tanto la T3 libre y total como T4 libre y total, se estabilizan los niveles de las mismas a rangos normales correspondientes en el momento de la remisión de la nefrosis.

\section{Conclusiones}

Con este trabajo se observó que los pacientes pediátricos que son diagnosticados con síndrome nefrótico, encontrándose en la fase activa del mismo (nefrosis), al realizarles pruebas de función tiroidea, el $90 \%$ presentan hipotiroidismo asociado, tomando en cuenta los niveles de la hormona estimulante de la tiroides. Se observó que el $78 \%$ de los niños con nefrosis se encuentran con hipotiroidismo subclínico y $10.5 \%$ con hipotiroidismo clínico, por lo que se concluye que es necesario realizar el perfil tiroideo a los pacientes con síndrome nefrótico en la nefrosis con mala respuesta al tratamiento inicial o recaídas frecuentes para poder identificar a los niños con hipotiroidismo asociado.

\section{Bibliografía}

1. Santos F. Síndrome nefrótico. Protocolos de nefrología. Boletín de la Sociedad de Pediatría de Asturias, Cantabria, Castillay 2006; 46(1): 19-23.

2. Román Ortiz E. Síndrome nefrótico pediátrico. Protocolo diagnostico terapéutico pediátrico. 2014; 1 : 283-301.

3. Schlesinger P. Sultz HJ. Mostherw PL. The nephrotic syndrome. Incidence and complicationes. Pediatric Nrphrology. 2013;12(8): 458-474.

4. Bagga A., Ali V., Banerjee S., Kanitkar M, Phadke $K D$, et al. Management of steroid sensitive nephrotic síndrome: Revised guideliness. Pediatric Indian. Review. 2008; 45(3):203-14

5. Comité de expertos en Nefrología. Síndrome nefrótico en niños. Bol Med Hosp Infant Mex. 2014. 57(9):522-536.

6. INEGI Instituto Nacional de Estadística y Geografía. https://www.inegi.org.mx/

7. ACCHMH Archivo Clínico del Centenario Hospital Miguel Hidalgo.

8. Schussler G.C. The thyroxine-binding proteins. Thyroid. 2000; (10): 141-149.

9. Peña Porta J. M., González J., De Vera Floristán C. V. Hypothyroidism subclinical and nephrotic síndro- 
me caused by membranous nephropathy. Nefrology 2008, 28 (5) 572-573

10. Kapoor K., Salía A., Goyal P. Subclinical non-autoinmune hypothyroidism in children with steroid resistant nephrotic síndrome. Clin exp, Nephrol. 2014 (18): 113-117.

11. Matto TK. Hypothyroidism in infants with nephotic síndrome. Pediatric Nephrology. 1994 Dec; 8(6): 503-15.

12. Ito S., Kano K., Ando T., Ichimura T. Thyroid function in children with nephrotic síndrome. Pediatric $\mathrm{Ne}$ phrology. 1994 Aug;8(4):412-5.

13. Katz Al, Emmanouel D.S., Lindheimer M.D., Thyroid hormone and the kidney. Nephron 1975; (15):223249.

14. Iglesias P. Diez J.J. Thyroid dysfuntion and kidney disease. Europeon Journal of Endocrinology. 2009 (160): 503-15

15. Afroz S., Khan A.H., Roy D.K. Thyroid function in children with nephrotic syndrome. Mymensingh Med J. 2011;20(3):407-11.

16. Vidhi S, Nanda S., Gehlawat V.K., Gathwala G. Hypothyroidism in Nephrotic Syndrome in Children. Journal of Dental and Medical Sciences. 2014;13(8): 7-11

17. Añez R. J., Morillo J., Rojas J, Rojas J, Torres Y, et al. Hipotiroidismo y su rara asociación con síndrome nefrótico: a propósito de un caso. Centro de Investigaciones Endocrino-Metabólicas. 2014; 4(1): 22-30.
18. Halty M. Caggiani M. Características evolutivas del síndrome nefrótico idiopático. Análisis de 70 casos. Archivos de pediatría del Uruguay. 2010;81(3):146157.

19. Fernández E. S. Monografía sobre el Síndrome Nefrótico. EUE. Casa de Salud Valdecilla. 2017;1: 1-27.

20. Kacer M., Whyte D.A., Boydstun I., Wilson TA. Congenital nephrotic síndrome and persistent hypothyroidism after bilateral nephrectomy Journal of Pediatrica Endocrinology \& Metabolism 2008; 21(6): 597-601.

21. Karethimmaiah H., Sarathi V. Nephrotic Syndrome Increases the Need for Levothyroxine Replacement in Patients with Hypothyroidism. Internal Medicine Section. 2016; 10(12):1-11.

22. Sharma, S., Kumar D. P., Kumar M. Evaluation of Thyroid Hormone Status in Children with Steroid Resistant Nephrotic Sydrome: A North Indian Study. Endocrine Metabolic \& Immune Disorders-Drugs Targets. 2015; 15(4):321-324.

23. Ebadi A., Shirali S., Daneghian S., Saki S. Evaluating the Thyroid Function in Pediatric Nephrotic Syndrome: A study conducted in Ahvaz, Iran. International Journal of Pharmaceutical Research \& Allied Sciences, 2016, 5(2):82-85.

24. Rondon Berrios H. Avances en la fisiopatología del edema en el síndrome nefrótico. Revista nefrología 2011;31(2):148-54. 